\title{
Clinical Features and Predictors of In-hospital Mortality in Patients with Acute and Chronic Pulmonary Thromboembolism
}

\author{
Norihisa KumasaKa, Masahito SaKuma and Kunio Shirato
}

\begin{abstract}
Objective The differences in the clinical findings of patients with acute pulmonary thromboembolism (acute-PTE) and chronic pulmonary thromboembolism (chronic-PTE) were compared, and the association between the clinical findings and prognosis in the two groups was analyzed.

Patients This study included 204 patients with PTE, 139 patients with acute-PTE and 65 patients with chronicPTE.

Results There were significant differences between acute-PTE and chronic-PTE in the predisposing factors of post operation (19.4 versus $1.5 \%, p=0.0003)$, and electrocardiographic signs such as sinus tachycardia $(73.7$ versus $50.9 \%, p=0.007$ ), ST-depression $(25.3$ versus $9.4 \%, p=0.03$ ), right ventricular hypertrophy $(20.0$ versus $47.2 \%$, $p=0.0007)$, and right axis deviation (3.2 versus $22.6 \%$, $p=0.0003$ ). Thirty-one of the 139 acute-PTE patients died from PTE, as did 17 of the 65 chronic-PTE patients. The prognosis was poor in patients older than 70 years old $(p=0.01)$, with stroke $(p=0.008)$, syncope $(p=0.01)$, shock $(p=0.0006)$, hypocapnia $\left(\mathrm{Paco}_{2} \leq 25\right.$ torr; $\left.p=0.0006\right)$ and an elevation in total pulmonary resistance (TPR $>1,000$ dyne sec $\left.\cdot \mathbf{c m}^{-5}(\mathbf{p = 0 . 0 2})\right)$ in acute-PTE, and in those with syncope $(p=0.03)$, shock $(p=0.008)$, and right ventricular hypertrophy on electrocardiogram $(\mathrm{p}=0.03)$ in chronic-PTE.

Conclusion The results of this study indicate a relationship between the clinical features of patients with acutePTE and chronic-PTE, and the predictors of in-hospital mortality.

(Internal Medicine 39: 1038-1043, 2000)
\end{abstract}

Key words: retrospective study, pulmonary hypertension

\section{Introduction}

In the United States, approximately 630,000 individuals suffer a pulmonary embolic event every year (1). Of these pa- tients, $90 \%$ survive the acute episode. In the vast majority, the emboli are resolved rapidly and substantially. However, in a subgroup with extensive embolization, the emboli fail to resolve and develop into chronic thromboembolic pulmonary hypertension (chronic-PTE with PH). The accession rate of patients with chronic-PTE with PH is $0.1 \%$ to $0.2 \%$ of survivors per year $(2,3)$. Our previous data confirmed that the incidence of PTE was low in Japan compared with that in the United States (4). However, little is known about the association between acute pulmonary thromboembolism (acute-PTE) and chronic pulmonary thromboembolism (chronic-PTE) among Japanese. There have never been detailed reports stating the differences between the clinical findings of Japanese patients with acute-PTE and chronic-PTE and the ratio of chronic-PTE to acute-PTE, or chronic-PTE with PH to acute-PTE. Therefore, we assessed the clinical, laboratory, radiographic, electrocardiographic, and hemodynamic findings in acute-PTE and chronic-PTE, and the association between the clinical factors and prognosis in the two groups.

\section{Methods}

We sent questionnaires to hospitals with a capacity of more than one hundred beds in the Tohoku region located in northern Japan, to inquire about the equipment of the hospitals and whether or not the doctors treated patients with PTE from 1985 to 1995 . Next, we examined the clinical records of patients when provided.

In this study, the diagnosis of PTE was confirmed by either 1) pulmonary artery stenosis or occlusion by pulmonary angiography, 2) mismatch of pulmonary perfusion scintigraphy and pulmonary ventilation scintigraphy, 3) changes on pulmonary perfusion scintigraphy performed twice, or 4) autopsies.

We classified the patients with PTE into two groups (acutePTE and chronic-PTE). We defined the chronic-PTE group as patients with an insidious onset (6 months to years) of symptoms (5) or patients with recurrent who suffered several unequivocal acute embolic episodes, and the patients without other pulmonary and heart disease who had more than $40 \mathrm{mmHg}$ in mean pulmonary artery pressure whatever the manner of onset

From the First Department of Internal Medicine, Tohoku University School of Medicine, Sendai

Received for publication January 25, 2000; Accepted for publication July 31, 2000

Reprint requests should be addressed to Dr. Kunio Shirato, the First Department of Internal Medicine, Tohoku University School of Medicine, 1-1 Seiryomachi, Aoba-ku, Sendai 980-8574 
as acute.

We reviewed the chest X-ray films and electrocardiogram at the onset of symptoms in the acute-PTE and recurrent type of chronic-PTE before treatment, and at admission in those with an insidious onset of chronic-PTE. On chest X-ray, an enlargement of the cardiac shadow was defined as a cardiothoracic ratio of 0.5 or more, and an enlargement of the pulmonary artery was defined as a right descending pulmonary artery of $20 \mathrm{~mm}$ or more, and hyperlucency (6) was determined by a marked reduction of a part of the pulmonary vessels. On electrocardiogram, sinus tachycardia was defined as an increase in sinus rhythm to $\geq 100$ beats/min. Right axis deviation (RAD) was defined as greater than 90 degrees in a mean frontal plane QRS electrical axis. Right bundle branch block (RBBB) was diagnosed when the terminal QRS forces were directed rightward and anteriorly, causing the production of $S$ wave in lead I and $R$ wave in lead $V_{1}$. Low $Q R S$ voltage was determined when the amplitude of the largest QRS deviation in the limb leads was $0.5 \mathrm{mV}$ or less. A pulmonary P wave was determined when its amplitude reached $0.25 \mathrm{mV}$ in lead II. The presence of $\mathrm{S}$ wave in lead I and Q wave in lead III, each with an amplitude exceeding $0.15 \mathrm{mV}$, associated with inversion of the T-wave in lead III constituted the $\mathrm{S}_{\mathrm{I}} \mathrm{Q}_{\mathrm{III}} \mathrm{T}_{\text {III }}$ pattern. Right ventricular hypertrophy $(\mathrm{RVH})$ was considered to be present if the $\mathrm{R}$ wave in lead $V_{1}$ exceeded $0.5 \mathrm{mV}$ or the $R / S$ ratio in lead $V_{1}$ was greater than one. ST-depression was considered present if the ST segment was depressed $0.05 \mathrm{mV}$ or more in any lead except $_{\mathrm{a}} \mathrm{V}_{\mathrm{R}}(7)$.

The systolic pulmonary artery pressure, diastolic pulmonary artery pressure, mean pulmonary artery pressure (PAm), and cardiac output (C.O) by dye-dilution or thermodilution method were measured by cardiac catheterization. Total pulmonary resistance (TPR) was calculated as follows: TPR (dyne $\cdot \mathrm{sec} \cdot$ $\left.\mathrm{cm}^{-5}\right)=(\mathrm{PAm}(\mathrm{mmHg}) / \mathrm{C} . \mathrm{O}(\mathrm{l} / \mathrm{min})) \times 80$. In the acute-PTE group and the recurrent type of the chronic-PTE group, the data of arterial blood gas, cardiac catheterization, pulmonary angiography, and perfusion scintigraphy were reviewed in patients who were treated before the thrombolytic or anticoagulant therapy and surgery. In the insidious onset type of the chronic-PTE group, the data on the admission in hospital were reviewed. The diagnosis of deep vein thrombosis was confirmed by venography of legs, but almost all patients had examination by venography after therapy such as by thrombolysis or anticoagulation.

Statistical analysis was carried out using the commercially available software of a statistical package (StatView ver 5.0). Values are presented as the mean + SD. Values in the two groups were compared by Fisher's exact test for dichotomous variables and the Wilcoxon rank test for continuous variables. The results of the logistic regression models are presented as estimated odds ratios with the corresponding $95 \%$ confidence intervals. The predictors of in-hospital death were investigated by logistic regression. All significance tests were two-tailed, with a value of $\mathrm{p}<0.05$ considered to indicate clinical significance.

\section{Results}

We received 197 replies out of 342 letters about the equipment of hospitals and patients with PTE. Therefore, the response rate was $57.6 \%$.

Based on the clinical records in the hospitals where patients with PTE were reported by the letters, the population was made up of 204 patients, 71 men and 133 women, with a mean age of $63.3 \pm 13.6$ years (range, 14 to 88 years) (Fig. 1). There was no seasonal variation of PTE in 189 patients whose onset of this disease was obvious (February and March, 29; April and May, 34; June and July, 31; August and September, 24; October and November, 37; December and January, 34 patients; $p=0.55$ ).

The study included 204 Japanese patients with PTE, among whom 139 patients with acute-PTE and 65 patients with chronic-PTE were confirmed. The ratio of patients with chronicPTE to acute-PTE was 0.47 . Table 1 gives the clinical characteristics of the two groups. There was no significant difference in age, sex, symptoms, and signs of physical examinations between acute-PTE and chronic-PTE group. Patients with acutePTE were older than those with chronic-PTE with PH (PAm $\geq 25 \mathrm{mmHg}$ ) (64 \pm 13 versus $58 \pm 15$ years, $\mathrm{p}=0.02)$. In 27 patients with acute-PTE the onset was after operation (major abdominal 11, orthopedic 4, gynecological 4, neurosurgical 4, urological 4 patients), and in one patient with chronic-PTE the onset was after orthopedic surgery. The patients with predisposing factors of post operation showed a significant difference between the two groups. Only 39 of 139 in acute-PTE, and 31 of 65 in chronic-PTE had venography of the legs.

On chest X-ray, the patients in the acute-PTE and chronicPTE groups had enlargement of the heart silhouette $(78.2 \%$ versus $73.1 \%, \mathrm{p}=0.60$ ), enlargement of the descending pulmonary artery $(67.3 \%$ versus $80.8 \%, p=0.11)$, and hyperlucency

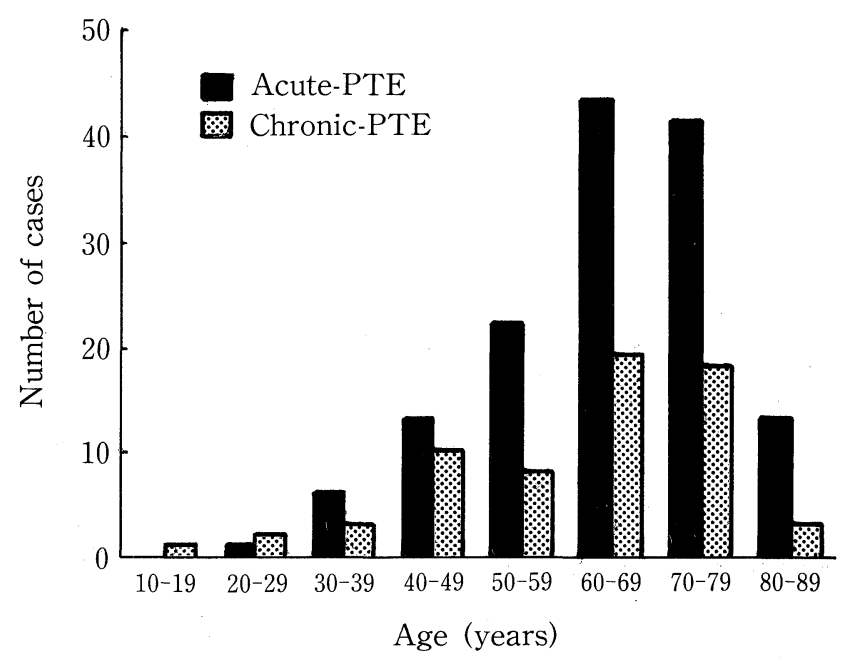

Figure 1. Distribution of disease by age in 204 patients with pulmonary thromboembolism (PTE). 
Table 1. Clinical Characteristics

\begin{tabular}{lccc}
\hline & APTE & CPTE & p Value \\
\hline Age, mean + SD & $64 \pm 15$ & $61 \pm 15$ & NS \\
Sex (male:female) & $47: 92$ & $24: 41$ & NS \\
BMI, mean \pm SD & $24.7 \pm 3.5$ & $22.6 \pm 3.3$ & $\mathrm{p}=0.07$ \\
Dyspnea & $113 / 126$ & $58 / 60$ & NS \\
Chest pain & $99 / 114$ & $43 / 54$ & NS \\
Syncope & $36 / 125$ & $18 / 57$ & NS \\
Shock & $55 / 132$ & $24 / 65$ & NS \\
Cardiac disease & $13 / 139$ & $4 / 65$ & NS \\
Post operation & $27 / 139$ & $1 / 65$ & p=0.0003 \\
Stroke & $16 / 139$ & $4 / 65$ & NS \\
DVT & $15 / 39$ & $10 / 31$ & NS \\
\hline
\end{tabular}

APTE: acute pulmonary thromboembolism, CPTE: chronic pulmonary thromboembolism, BMI: body mass index, Shock: systolic blood pressure $<90 \mathrm{mmHg}$, DVT: deep vein thrombosis, NS: not significant.

(35.5\% versus $38.5 \%, \mathrm{p}=0.84)$. On electrocardiogram, in the patients with acute-PTE, T-wave inversion in $\mathrm{V}_{1-3}(81.1 \%)$ and sinus tachycardia $(73.7 \%)$ were the most common electrocardiographic signs. On the other hand, in the patients with chronicPTE, T-wave inversion in $\mathrm{V}_{1-3}(75.5 \%)$ was found. Sinus tachycardia, ST-depression, right ventricular hypertrophy, and right axis deviation were significantly different between the two groups (Table 2). In acute-PTE, there was a significant relationship between patients with ST-depression and shock (systolic blood pressure $<90 \mathrm{mmHg}$ ) ( $\mathrm{p}=0.02$ ), but not between ST-depression and pulmonary artery pressure, and hypoxemia.

One hundred-nine of the 139 patients with acute-PTE and 53 of the 65 patients with chronic-PTE had arterial blood gas analysis. Fifty-three of the 139 patients with acute-PTE and 46 of the 65 patients with chronic-PTE had cardiac catheterization. Thirty-eight of the 65 patients $(58.5 \%)$ with chronic-PTE had pulmonary hypertension (PAm $\geq 25 \mathrm{mmHg}$ ), and 17 of 65 patients accorded in the criteria for chronic-PTE with PH from the Japanese Ministry of Health and Welfare. The pulmonary artery pressure and total pulmonary resistance in the chronicPTE group were higher than those in acute-PTE (Table 3).

There were no significant differences between acute-PTE and chronic-PTE groups in the defects of segment by pulmonary angiography and perfusion scintigraphy. The patients in the acute-PTE and chronic-PTE groups were treated with thrombolytic therapy by urokinase ( 46.8 versus $44.6 \%, p=0.88$ ) or tissue plasminogen activator ( 16.5 versus $12.3 \%, \mathrm{p}=0.53$ ), heparin $(66.2$ versus $52.3 \%, \mathrm{p}=0.06)$, warfarin $(62.6$ versus $70.8 \%, \mathrm{p}=0.27)$, intervention therapy $(7.2$ versus $1.5 \%, \mathrm{p}=0.18)$, or surgical treatment $(0.7$ versus $1.5 \%, \mathrm{p}=0.54)$.

In-hospital deaths due to PTE were 31 of the 139 in the acute-PTE group, and 17 of the 65 in the chronic-PTE group $(\mathrm{p}=0.60)$. Over 70 years old, stroke, syncope, shock, hypocapnia $\left(\mathrm{Paco}_{2} \leq 25\right.$ torr), and more than 1,000 dyne $\cdot \mathrm{sec} \cdot \mathrm{cm}^{-5}$ in total pulmonary resistance were associated with mortality in
Table 2. Electrocardiographic Signs

\begin{tabular}{lccc}
\hline & $\begin{array}{c}\text { APTE }(\mathrm{n}=95) \\
(\%)\end{array}$ & $\begin{array}{c}\text { CPTE }(\mathrm{n}=53) \\
(\%)\end{array}$ & $\mathrm{p}$ Value \\
& $77(81.1)$ & $40(75.5)$ & $\mathrm{NS}$ \\
${\text { T inversion in } \mathrm{V}_{1-3}}$ & $70(73.7)$ & $27(50.9)$ & $\mathrm{p}=0.007$ \\
Sinus tachycardia & $36(37.9)$ & $19(35.8)$ & $\mathrm{NS}$ \\
$\mathrm{S}_{\mathrm{I}} \mathrm{Q}_{\mathrm{III}} \mathrm{T}_{\mathrm{III}}$ & $31(32.6)$ & $15(28.3)$ & $\mathrm{NS}$ \\
RBBB & $24(25.3)$ & $5(9.4)$ & $\mathrm{p}=0.03$ \\
ST depression & $19(20.0)$ & $25(47.2)$ & $\mathrm{p}=0.0007$ \\
RVH & $20(21.1)$ & $17(32.1)$ & $\mathrm{NS}$ \\
Pulmonary P & $18(18.9)$ & $15(28.3)$ & $\mathrm{NS}$ \\
Clockwise rotation & $3(3.2)$ & $12(22.6)$ & $\mathrm{p}=0.0003$ \\
RAD & $5(5.3)$ & $8(15.1)$ & $\mathrm{p}=0.07$ \\
Low voltage & $3(3.2)$ & $1(19)$ & $\mathrm{NS}$ \\
ST elevation in $\mathrm{V}_{1-3}$ & & & \\
\hline
\end{tabular}

RBBB: right bundle branch block, RVH: right ventricular hypertrophy, RAD: right axis deviation, Other abbreviations see Table 1.

Table 3. Hemodynamic Data

\begin{tabular}{lccc}
\hline & APTE & CPTE & $\mathrm{p}$ Value \\
\hline Arterial blood gas & $7.44 \pm 0.06$ & $7.42 \pm 0.08$ & $\mathrm{p}=0.06$ \\
$\mathrm{pH}$ & $56.7 \pm 12.2$ & $58.5 \pm 10.7$ & $\mathrm{NS}$ \\
$\mathrm{Pao}_{2}$ (torr) & $32.1 \pm 6.9$ & $33.4 \pm 8.7$ & $\mathrm{NS}$ \\
$\mathrm{Paco}_{2}$ (torr) & & & \\
Cardiac catheterization & & & \\
PAs (mmHg) & $44 \pm 14$ & $71 \pm 27$ & $\mathrm{p}<0.0001$ \\
$\mathrm{PAd}(\mathrm{mmHg})$ & $17 \pm 6$ & $27 \pm 12$ & $\mathrm{p}<0.0001$ \\
$\mathrm{PAm}(\mathrm{mmHg})$ & $27 \pm 8$ & $43 \pm 17$ & $\mathrm{p}<0.0001$ \\
$\mathrm{C} . \mathrm{O}(\mathrm{l} / \mathrm{min})$ & $3.92 \pm 1.34$ & $4.09 \pm 1.44$ & $\mathrm{NS}$ \\
TPR (dyne $\left.\cdot \mathrm{sec} \cdot \mathrm{cm}^{-5}\right)$ & $671 \pm 394$ & $950 \pm 487$ & $\mathrm{p}=0.03$ \\
\hline
\end{tabular}

A total of 109 with APTE and 53 with CPTE underwent arterial blood gas analysis, and 53 with APTE and 46 with CPTE had cardiac catheterization. Data presented are mean $\mathrm{SD}$, PAs: systolic pulmonary artery pressure, PAd: diastolic pulmonary artery pressure, PAm: mean pulmonary artery pressure, C.O: cardiac output, TPR: total pulmonary resistance. Other abbreviations see Table 1.

acute-PTE, and syncope, shock, and right ventricular hypertrophy on electrocardiogram in chronic-PTE (Table 4).

\section{Discussion}

Our data indicated that there are some differences in several clinical findings including predisposing factors, electrocardiogram, hemodynamics, and predictors in-hospital mortality between the acute-PTE and chronic-PTE groups, and that the ratio of patients with chronic-PTE to acute-PTE is higher in Japan than in the United States.

\section{Clinical features}

There was a significant difference between acute-PTE and 
Pulmonary Thromboembolism in Japan

Table 4. Predictors of In-hospital Death

\begin{tabular}{|c|c|c|}
\hline & $\begin{array}{c}\text { APTE } \\
\text { OR }(\mathrm{CI}), \mathrm{p} \text { value }\end{array}$ & $\begin{array}{c}\text { CPTE } \\
\text { OR (CI), p value }\end{array}$ \\
\hline Sex (female) & $1.33(0.56-3.17), p=0.52$ & $1.57(0.48-5.18), p=0.46$ \\
\hline Age $\geq 70$ & $2.77(1.22-6.28), p=0.01$ & $2.39(0.76-7.53), p=0.14$ \\
\hline Dyspnea & $0.88(0.16-4.13), p=0.79$ & - \\
\hline Chest pain & $0.95(0.24-3.70), \mathrm{p}=0.94$ & $0.60(0.15-2.46), p=0.48$ \\
\hline Syncope & $3.03(1.25-7.36), \mathrm{p}=0.01$ & $3.88(1.16-12.96), \mathrm{p}=0.03$ \\
\hline Shock & $4.74(1.96-11.44), p=0.0006$ & $4.94(1.52-16.08), p=0.008$ \\
\hline Cardiac disease & $1.63(0.47-5.70), p=0.44$ & $0.94(0.09-9.68), \mathrm{p}=0.96$ \\
\hline Post operation & $0.23(0.05-1.03) \mathrm{p}=0.054$ & - \\
\hline Stroke & $4.35(1.48-12.80), p=0.008$ & $10.07(0.97-104.63), p=0.053$ \\
\hline DVT & - & $2.25(0.27-18.30), p=0.46$ \\
\hline $\mathrm{T}$-wave inversion in $\mathrm{V}_{1-3}$ & $0.81(0.23-2.83), \mathrm{p}=0.74$ & $0.40(0.09-1.72), p=0.22$ \\
\hline Sinus tachycardia & $1.20(0.35-4.09), \mathrm{p}=0.77$ & $0.96(0.24-3.78), p=0.95$ \\
\hline $\mathrm{S}_{\mathrm{I}} \mathrm{Q}_{\mathrm{III}} \mathrm{T}_{\mathrm{III}}$ & $2.21(0.77-6.37), p=0.14$ & $0.38(0.07-2.02), p=0.26$ \\
\hline RBBB & $1.63(0.56-4.80), \mathrm{p}=0.37$ & $0.58(0.11-3.10), p=0.52$ \\
\hline ST depression & $1.88(0.61-5.77), p=0.27$ & $1.08(0.11-10.90), \mathrm{p}=0.95$ \\
\hline $\mathrm{RVH}$ & $0.86(0.22-3.35), \mathrm{p}=0.82$ & $6.12(1.16-32.36), \mathrm{p}=0.03$ \\
\hline RAD & $10.53(0.90-123.70), p=0.06$ & $0.32(0.37-2.85), \mathrm{p}=0.31$ \\
\hline $\mathrm{PaO}_{2} \leq 50$ (torr) & $0.61(0.20-1.85), p=0.38$ & $1.59(0.34-7.39), p=0.55$ \\
\hline $\mathrm{PaCO}_{2} \leq 25$ (torr) & $8.31(2.52-27.44), \mathrm{p}=0.0006$ & $1.30(0.22-7.54), p=0.77$ \\
\hline $\mathrm{PAm} \geq 25(\mathrm{mmHg})$ & - & $0.55(0.08-3.68), \mathrm{p}=0.54$ \\
\hline $\mathrm{TPR}>1,000\left(\right.$ dyne $\left.\cdot \mathrm{sec} \cdot \mathrm{cm}^{-5}\right)$ & $19.0(1.45-248.30), \mathrm{p}=0.02$ & $0.50(0.74-3.38), \mathrm{p}=0.48$ \\
\hline
\end{tabular}

In the arterial blood gas analysis, the number of patients with $\mathrm{Pao}_{2} \leq 50$ torr was 37 with APTE and 11 with $\mathrm{CPTE}$, and the number of patients with $\mathrm{Paco}_{2} \leq 25$ torr was 15 with APTE and 8 with CPTE. In the cardiac catheterization data, the number of patients with PAm $\geq 25 \mathrm{mmHg}$ was 35 with APTE and 38 with CPTE, and the number of patients with TPR $>1,000 \mathrm{mmHg}$ was 6 with APTE and 12 with CPTE. For other abbreviations and the number of patients with each predictor, see Tables 1-3. OR: odds ratio, Cl: confidence intervals. We could not calculate the odds ratio for several factors, because no patient who had deep vein thrombosis and less than $25 \mathrm{mmHg}$ pulmonary artery pressure had died in acute-PTE, and no patient who had symptoms of dyspnea and post operation had died in chronic-PTE.

chronic-PTE group in post operation as a predisposing factor. Long-term immobility and hypercoagulation after operation result in large venous thrombi that subsequently develop into acute-PTE, whereas the establishment of chronic-PTE appears to be associated with recurrent emboli and their incomplete resolution, but not with acute changes in the patient's load such as post operation. Okada et al (8) reported that the HLA pattern in chronic-PTE with PH is similar to that in aortitis syndrome. It is of interest that there was a difference between acutePTE and chronic-PTE in the mechanism of emboli. A prevention of PTE after operation has been done in Western countries, and in the future such prevention should become important in Japan. Moreover, most cases with acute-PTE were induced by deep vein thrombosis in Western countries (9), but deep vein thrombosis was not found in so many patients with acute-PTE in the present study. This is similar to the report of Kunieda et al (10). They indicated that the contribution of deep vein thrombosis to acute-PTE is different between Japan and the United States. We showed that the body mass index of patients with acute-PTE tended to be higher than that with chronicPTE. The result may agree with a previous report that obesity is one of the riskfactors for acute-PTE (11).

On electrocardiogram, there were more patients with sinus tachycardia and ST-depression with acute-PTE than with chronic-PTE. As reported by Petruzzelli et al (12), these signs are the most common findings in patients with suspected acutePTE. The marked increase in resistance against blood flow through the lungs can result in a significant decrease in cardiac output and tachycardia (13). In the present study, despite the absence of differences in cardiac output between acute-PTE and chronic-PTE, sinus tachycardia occurs more frequently in acute-PTE than in chronic-PTE, and it was not possible to establish a definite causal relationship between sinus tachycardia and cardiac output by cardiac catheterization in acute-PTE. However, although the reason for these results is not clear, the first possible explanation is that the time of performing cardiac catheterization was not consistent after the onset of PTE in each case, and the second is that the neurohumoral changes might differ between the two groups. ST-depression has been thought to be related to right ventricular pressure overload or myocardial ischemia due to shock and hypoxemia secondary to PTE. Although we found no relationship between patients 
with ST-depression and hypoxemia, and pulmonary artery pressure, there was a significant relationship between patients with ST-depression and shock. On the other hand, in chronic-PTE the electrocardiogram showed right heart overload, such as right ventricular hypertrophy and right axis deviation. These findings seem to be a compensatory mechanism against the higher pulmonary vascular resistance in chronic-PTE than in acutePTE. These electrocardiographic findings may be useful to distinguish acute-PTE from chronic-PTE.

\section{Predictors of in-hospital death}

In the present study, shock and syncope predicted in-hospital mortality in acute-PTE and chronic-PTE, and an age greater than 70 years old, stroke, hypocapnia, and elevated total pulmonary resistance also were related to in-hospital death in acutePTE. The presence of concomitant diseases and older age has been thought to be the most important factors. Carson et al (14) reported that over 60 years indicated a poor prognosis in acute-PTE, because many old patients usually have coronary artery or cerebral diseases, and the existence of failing and ischemic myocardium can especially have an adverse outcome. The present results are consistent with their report concerning the risk factors of advanced aged and the concomitant presence of stroke. Moreover, hypocapnia caused by hyperventilation in acute-PTE was relevant to the prognosis, and extensive hyperventilation may show massive acute-PTE. Goldhaber et al (15) showed that tachypnoea was correlated with a poor prognosis in acute-PTE. Moreover, in the acute phase, the extent of pulmonary vascular obstruction reflected by the degree of pulmonary hypertension has been thought to be one of the prognostic factors (16). The prognosis was not associated with the pulmonary artery pressure but with an elevation of total pulmonary resistance. As the reason for this result we considered that total pulmonary resistance more precisely reflected the degree of occlusion in the pulmonary vasculature than the pulmonary artery pressure. In this study, our interest was directed to predictors associated with short-term in-hospital mortality in chronic-PTE. In the report of Riedel et al (17), the prognosis was serious in all patients with a mean pulmonary artery pressure over $30 \mathrm{mmHg}$ in chronic-PTE with $\mathrm{PH}$. Although there were 34 patients with a mean pulmonary artery pressure over $30 \mathrm{mmHg}$ in this study, there was no relationship between mean pulmonary artery pressure and the prognosis. This does not mean that pulmonary hypertension as a predictor of prognosis is controversial, because our predictor implied short-term inhospital mortality, whereas Riedel et al assessed long-term predictors. While right ventricular hypertrophy on electrocardiogram was significantly related to short-term in-hospital mortality, we think this reflects the extent of right heart failure.

\section{Acute-PTE and chronic-PTE with PH}

After most patients with chronic-PTE with PH had the initial acute embolic event and deep vein thrombosis, they had a symptom free period, that is a "honeymoon" period. But after the period, they increasingly suffered from dyspnea on exertion. As it is assumed that the patients with acute-PTE are most likely to develop chronic-PTE with PH in Western countries (2), we classified the patients with PTE into two groups by their histories and clinical features in this study. The prevalence of chronic-PTE with PH was $0.1-0.2 \%$ of acute-PTE in the United States (3), and $0.14 \%$ over the years 1966-1990 (18). In the present study, the ratio of Japanese patients with chronic-PTE and with both chronic-PTE and PH to acute-PTE was 0.47 and 0.27 , respectively, which are higher ratios than in United States. Several reasons for the difference are considered. First, the correct diagnostic rate of patients with PTE was as low as $23 \%$ in Japan (10), so that some patients would have unsuccessful lysis of acute-PTE because of the late start of therapy. Second, we think that the origin of chronic-PTE with PH may be differ according to race. For example, the number of male cases was lower than that of females in our previous data (4), and the ratio of males to females was similarly low in this study, while the incidence of chronic-PTE with PH was higher in males than in females in United States (19). In this study, patients with chronic-PTE with PH were younger than patients with acute-PTE, consistent with the report by Kunieda (20). Therefore, it is unlikely in most cases in Japan that the patients with acute-PTE develop chronic-PTE with PH.

We sent questionnaires not only to the hospitals with special departments such as cardiology and respiratory medicine but also to the hospitals of general medicine, and we think the results reflect the actual conditions of patients with PTE in Japan. We retrospectively investigated the clinical features of the patients with PTE by clinical records, and classified the patients with PTE into two groups. One of the limitations in the present study is incompleteness of the clinical records in some cases. In almost all of our patients, deep vein thrombosis had been assessed after thrombolitic or anticoagulant therapy. This seems to have affected the incidence of deep vein thrombosis in the present study, which was lower than that in the United States. Moreover, although the Japanese Ministry of Health and Welfare has set up some definite criteria for chronic-PTE with PH, we had no clear criteria in 1995, and our subjects were patients from 1985 to 1995 . At that time, general physicians had generally employed the definition of chronic-PTE as mentioned in methods. The classification of PTE has been controversial among investigators. Therefore, we simply classified PTE into two categories (acute-PTE and chronic-PTE). Precise definitions will be needed to examine the detailed profiles of the patients with PTE. In Japanese patients with acutePTE and chronic-PTE, we investigated predisposing factors, electrocardiographic, and hemodynamic findings, and the association between clinical factors and prognosis. These features seem to be very important for the diagnosis and therapy of the patients with PTE, and we consider that a prospective study is necessary to confirm the present results.

Acknowledgements: We would like to thank the doctors who contributed to this study. 


\section{Pulmonary Thromboembolism in Japan}

\section{References}

1) Dalen JE, Alpert JS. Natural history of pulmonary embolism. Prog Cardiovasc Dis 17: 257-270, 1975.

2) Moser KM, Auger WR, Fedullo PF. Chronic major-vessel thromboembolic pulmonary hypertension. Circulation 81: 1735-1743, 1990.

3) Jamieson SW, Auger WR, Fedullo PF, et al. Experience and results with 150 pulmonary thromboendarterectomy operations over a 29 -month period. J Thorac Cardiovasc Surg 106: 116-127, 1993.

4) Kumasaka N, Sakuma M, Shirato K. Incidence of pulmonary thromboembolism in Japan. Jpn Circ J 63: 439-441, 1999.

5) Ansari A. Acute and chronic pulmonary thromboembolism. Current perspectives. Part I . Glossary of Terms, historic evolution and prevalence. Clin Cardiol 9: 398-402, 1986.

6) Palla A, Giuntini C. Imaging of pulmonary embolism. in: Lung Biology in Health and Disease, vol 75. Pulmonary Embolism, Morpurgo M, Ed. Marcel Dekker, Inc. New York, 1994: 115-151.

7) Stein PD, Dalen JE, Mclntyre KM, Sasahara AA, Wenger NK, Willis PW. The electrocardiogram in acute pulmonary embolism. Prog Cardiovasc Dis 17: 247-257, 1975.

8) Okada O, Tanabe N, Yasuda J, et al. Comprehensive study for genetic factors in chronic thromboembolic pulmonary hypertension. in: Annual report of the study project of respiratory failure research committee supported by Japanese Ministry of Health and Welfare Research Grant 1997. K Planning Inc., 1998; 140-142 (in Japanese, English translation by present authors)

9) Hirsh J, Hoak J. Management of deep vein thrombosis and pulmonary embolism. A statement for healthcare professionals. Council on Thrombosis (in consultation with the Council on Cardiovascular Radiology),
American Heart Association. Circulation 93: 2212-2245, 1996.

10) Kunieda T, Naito M, Ookubo S, Yoshioka T, Nakanishi N, Oobayashi Y. Clinical aspects and diagnosis of pulmonary thromboembolism. (in Japanese) Jpn J Thorac Dis 26: 463-472, 1988.

11) Goldhaber SZ, Savage DD, Garrison RJ, et al. Risk factors for pulmonary embolism. The Framingham Study. Am J Med 74: 1023-1028, 1983.

12) Petruzzelli S, Palla A, Pieraccini F, Donnamaria V, Giuntini C. Rountine electrocardiography in screening for pulmonary embolism. Respiration 50: $233-243,1986$.

13) Schlant RC. Cardiac manifestations of pulmonary embolism. in: Lung Biology in Health and Disease, vol 75. Pulmonary Embolism, Morpurgo M, Ed. Marcel Dekker, Inc. New York, 1994: 67-78.

14) Carson JL, Kelley MA, Duff A, et al. The clinical course of pulmonary embolism. N Engl J Med 326: 1240-1245, 1992.

15) Goldhaber SZ, Visani L, De Rosa M. Acute pulmonary embolism: clinical outcomes in the International Cooperative Pulmonary Embolism Registry (ICOPER). Lancet 353: 1386-1389, 1999.

16) Widimsky J. Prognosis of pulmonary embolism. in: Lung Biology in Health and Disease, vol 75. Pulmonary Embolism, Morpurgo M, Ed. Marcel Dekker, Inc. New York, 1994: 97-105.

17) Riedel M, Stanek V, Widimsky J, Prerovsky I. Longterm follow-up of patients with pulmonary thromboembolism. Chest 81: 151-158, 1982.

18) Silverstein MD, Heit JA, Mohr DN, Petterson TM, O'Fallon WM, Melton III LJ. Trends in the incidence of deep vein thrombosis and pulmonary embolism. Arch Intern Med 158: 585-593, 1998.

19) Archibald CJ, Auger WR, Fedullo PF, et al. Long-term outcome after pulmonary thromboendarterectomy. Am J Respir Crit Care Med 160: 523528, 1999.

20) Kunieda T. Pulmonary arterial thromboembolism. Present status of epidemiology in Japan. Resp Circ 37: 325-332, 1989 (in Japanese). 